\title{
Arzneipflanzen, Inhaltsstoffe und Anwendung: Status quo und Entwicklungsmöglichkeiten
}

\author{
Bericht zum 29. Bernburger Winterseminar
}

\begin{abstract}
Am 19. und 20. Februar 2019 fand wie auch in den vergangenen Jahren auf Einladung der Saluplanta e.V. in der Landesanstalt für Landwirtschaft und Gartenbau Sachsen-Anhalt in Bernburg-Strenzfeld das Bernburger Winterseminar statt, an dem sich mehr als 250 Teilnehmer über neue Erkenntnisse aus dem Anbau, aus Züchtungsund Inhaltsstoffforschung sowie zu Qualitätsanforderungen und Anwendungsmöglichkeiten von Arznei- und Gewürzpflanzen informierten und ihre Erfahrungen austauschten.
\end{abstract}

Nach der Begrüßung durch die Organisatoren der Veranstaltung Dr. Bernd Hoppe, Bernburg, und Dr. Wolfram Junghanns, Aschersleben, sowie den Grußworten des Staatssekretärs Dr. Ralf-Peter Weber, Ministerium für Umwelt, Landwirtschaft und Energie des Landes Sachsen-Anhalt, gab Dr.-Ing. Andreas Schütte, Gülzow-Prüzen, einen Überblick über die von der Fachagentur Nachwachsende Rohstoffe e. V. (FNR) seit ihrer Gründung im Jahr 1993 geförderten 130 Forschungsvorhaben im Bereich der Arzneiund Gewürzpflanzen in einer Größenordnung von 26 Mio. Euro. Die Förderung umfasse Projekte aus z.B. Züchtung, Anbauoptimierung, Unkrautregulierung, Erntetechnologie und Trocknung, die u. a. Bestandteil des 2007 gestarteten Demonstrationsvorhaben mit den Beispielkulturen Kamille, Melisse und Baldrian mit 35 Teilprojekten und einer Fördersumme von 8,2 Mio. Euro seien, was einer Förderquote von 90 \% entspreche.

\section{Qualitätsanforderungen und rechtliche Einordnung}

Prof. Dr. Bernd Honermeier, Gießen, beschrieb aus Sicht der Forschung Aspekte der Qualität und Produktion von Arznei- und Gewürzpflanzen, insbesondere Anforderungen an Böden, Saat- und

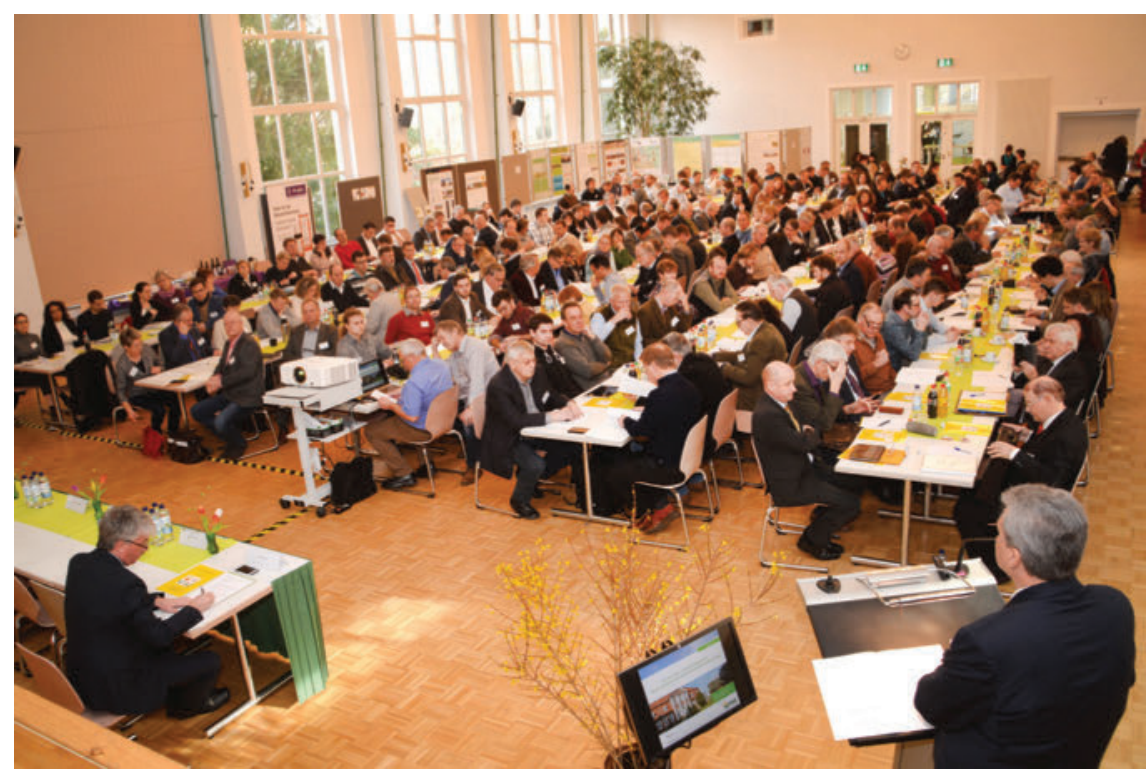

-Abb. 1 Volles Haus in Bernburg: Blick in den Tagungssaal. Foto: Karin Hoppe, Bernburg

Pflanzgut, Düngung, Pflanzenschutzmaßnahmen, Bewässerung, Ernte und Trocknung. Zur Optimierung der Qualität sei eine Erweiterung der Kenntnisse über wertgebende, aber auch toxische Inhaltsstoffe, Rückstände und Kontaminanten, Standorteignung und Anbaumanagement wichtig, ebenso zur Interaktion der Pflanze mit der Umwelt besonders unter dem Aspekt des Klimawandels und seiner Folgen. Er nannte als Beispiele die Ergebnisse von Forschungsarbeiten, die sich mit dem Screening der Ätherisch-Öl-Gehalte in Origanum-Arten oder Pfefferminze befassten. Untersuchungen zur vertikalen Verteilung des HypericinVorkommens in Johanniskrautpflanzen gäben wertvolle Hinweise zur Festlegung der Breite der zu erntenden Blühhorizonte, ähnliche Phänomene seien bei Buchweizen oder beim Vorkommen der Kaffeoylchinasäure-Gehalte in Artischockenblättern zu beobachten. Auch die optimale Nährstoffversorgung sei eine zukunftsweisende Aufgabe, wie z.B. die Düngung mit lebenden Mikroben als „Biofertilizer“.
Welche Grenzwerte der Gesetzgeber im Sinne des Verbraucherschutzes und der Patientensicherheit für unerwünschte, potenziell gesundheitsschädliche Begleitstoffe in pflanzlichen Materialien festgelegt hat, stellte Dr. Barbara Steinhoff, Bonn, dar. Sie erläuterte die praktische Relevanz dieser Regelungen für Schwermetalle bzw. Elementverunreinigungen, Pflanzenschutzmittel, polyzyklische aromatische Kohlenwasserstoffe (PAK) und Pyrrolizidinalkaloide (PA) sowie die große Bedeutung der Erhebung entsprechender Daten in der täglichen Praxis der pharmazeutischen Unternehmen im Rahmen eines firmenspezifischen / -übergreifenden Monitorings. Die Auswertung von Daten für die genannten Rückstände bzw. Kontaminanten biete einen umfassenden Überblick über die Belastungssituation und gebe den Unternehmen die Möglichkeit einer Risikoabschätzung, die auch eine Voraussetzung für die individuelle Gestaltung der Prüffrequenzen für die pflanzlichen Wirkstoffe bzw. Produkte sei. Letztendlich sei auch die auf Daten basierende Prüfung der Einhaltbarkeit von 
Grenzwerten eine wichtige Argumentationsgrundlage zur Diskussion mit den Behörden, wie auch die neuesten Entwicklungen am Beispiel PA gezeigt hätten.

Prof. Dr. Michael Keusgen, Marburg, untersuchte die Frage, wann eine Arzneipflanze als Arzneimittel und wann als Nahrungsergänzungsmittel einzustufen ist, da bekanntermaßen sekundäre Pflanzeninhaltsstoffe und hochkonzentrierte Extrakte teilweise auch als Nahrungsergänzung angeboten würden. Letztere seien allerdings nur verkehrsfähig, wenn sie keine pharmakologische Wirkung entfalteten bzw. nach aktueller Rechtsprechung die „Erheblichkeitsschwelle“ nicht überschritten werde. Er nannte als Beispiel Zubereitungen aus der Rosenwurz (Rhodiola rosea); hingegen seien die chinesischen Heilpilze Cordyceps sinensis und Ganoderma lucidum eher als „Novel Food“ einzustufen. Im Lebensmittelbereich verwendete Extrakte bräuchten keine Arzneimittelstandards zu erfüllen, allerdings werde in der Werbung häufig auf Ergebnisse analoger pflanzlicher Arzneimittel Bezug genommen. Die Rechtslage in Europa sei uneinheitlich, und der Verbraucher könne die Produktgruppen meistens nicht unterscheiden. Vorsicht sei jedenfalls geboten bei Internet-Käufen von Produkten mit Gesundheitsbezug. Der Referent betonte, dass Nahrungsergänzungsmittel Phytopharmaka sicher nicht ersetzen könnten.

\section{Pflanzen aus anderen Kulturkreisen}

Prof. Dr. Michael H. Böhme, Berlin, sprach über Pflanzen in der traditionellen asiatischen Medizin anhand der Länderbeispiele Bangladesch und Vietnam, in denen sowohl die Richtungen des indischen Ayurveda und des auf den Erkenntnissen der griechischen Medizin zu Zeiten von Hippokrates beruhenden Unani besonders ausgeprägt seien. Beide nutzten in der Therapie indigene Pflanzen wie das Indische Lungenkraut (Adhatoda vasica), den Ingwer (Zingiber officinale), das Indische Basilikum (Ocimum sanctum) oder die Schlafbeere (Withania somnifera). Der Referent

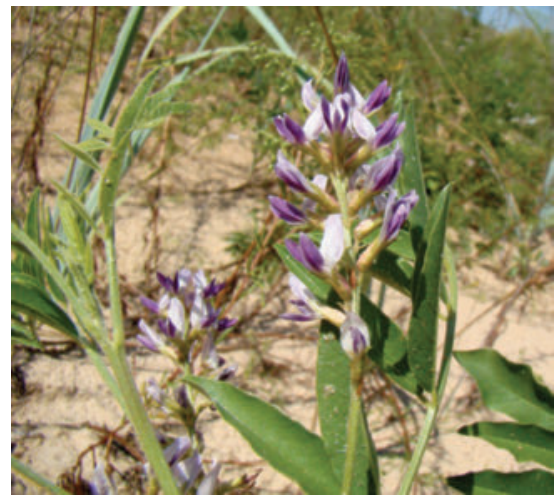

-Abb.2 Süßholzwurzel (Glycyrrhiza glabra): Guter Wurzelertrag auch bei Niederschlagsarmut im Iran. Foto: Yuriy Danilevsky

beschrieb außerdem den therapeutischen Wert einiger weiterer Pflanzen, die vorwiegend als Gewürze bzw. Nahrungsmittel eingesetzt werden, wie z. B. die polyphenolund anthocyanhaltige Lamiacee Perilla frutescens.

Ein weiterer Vortrag befasste sich mit der Domestizierung und dem Versuchsanbau von Süßholz (Glycyrrhiza glabra) im Iran, vorgetragen von Dr. Farsad Nadjafi, Teheran. Er zeigte, dass bei geringen Niederschlagsmengen ein guter Wurzelertrag erreicht werden konnte, der mit dem Gehalt an Glycyrrhizin und Glabridin als wertgebende Inhaltsstoffe korreliere. Ein großes, aber bislang nicht genutztes Potenzial zur weiteren Entwicklung von Arznei- und Gewürzpflanzen biete die Flora der Türkei, wie Prof. Dr. Mensure Özgüven, Konya / Türkei, darstellte. Durch eine staatliche Unterstützung sei es in den letzten Jahren möglich gewesen, Anbau und Verarbeitung von Arzneipflanzen voranzubringen sowie traditionelle volksmedizinische und ethnobotanische Kenntnisse wissenschaftlich auszuwerten und in die Vermarktung von Produkten umzusetzen. Einen umfassenden Überblick über Inhaltsstoffe, Forschungsarbeiten und Anwendungsmöglichkeiten des Schmalblättrigen Weidenröschens (Epilobium angustifolium) gab Prof. Dr. Elena Malankina, Universität Moskau, die berichtete, dass für diese Pflanze eine Inkulturnahme in verschiedenen Regionen Russlands geplant ist.

\section{Arzneipflanzen und Umwelt}

Dr. Matthias Lorenz, Darmstadt, stellte interessante Möglichkeiten für die Nutzung von Satellitendaten für das Sourcing von Arzneipflanzen dar. Als Beispiele für eine Optimierung der Qualitätssicherung erwähnte er den Kamillenanbau in Oberägypten, bei dem mit Hilfe von GPS-Daten eine Bewässerung aus sauberen Kanälen zur Vermeidung einer mikrobiellen Kontamination durchgeführt wurde, sowie die Gewinnung von Rotem Weinlaub in Chile, bei dem auf die gleiche Weise das Risiko einer Kontamination mit Pestiziden als Folge einer Windverwehung minimiert werden konnte. Anschaulich erläuterte er auch, wie potenzielle neue Sammelgebiete für Johanniskraut (Hypericum perforatum) in Chile, die durch Satellitenbilder sichtbar gemacht werden konnten, und wie schließlich im Rahmen von Forschungs- und Entwicklungsprojekten eine Verbesserung der Qualität von Muskatnüssen (Myristica fragans) mit Hilfe eines genetischen Screenings natürlicher Vorkommen in Grenada erreicht werden konnte.

Dr. Wolfram Junghanns beschrieb eindrücklich die katastrophalen Auswirkungen der Dürreperiode, die insbesondere in der zweiten Jahreshälfte 2018 den Arzneipflanzenanbau vor allem in Mitteldeutschland hochgradig beeinträchtigt hat. Durch extreme Trockenheit und starke Hitze sei es in vielen Kulturen zu Totalausfällen in der Ernte gekommen, wobei auch teilweise bereits in den frühen Stadien der Arzneipflanzenproduktion eine Denaturierung im Saatgut stattgefunden habe. Um zukünftigen Extremsituationen begegnen zu können, sei es deshalb notwendig, systematisch alle vorhandenen Bewässerungsmöglichkeiten auszuschöpfen und eine ernteübergreifende Bevorratung mit Rohware sicherzustellen, was allerdings kapitalintensiv sein und das Endprodukt verteuern könne.

Weitere Vorträge befassten sich u.a. mit Benediktenkraut (Centaurea benedicta) und seinen Bitterstoffen, mit der Züchtung von Johanniskraut (Hypericum perforatum), mit 
dem Auftraten von Schaderregern in Kamille (Matricaria recutita) sowie mit neuen Anwendungsregelungen für Düngemittel und Pflanzenschutzmittel.

\section{Ehrungen und \\ Rahmenprogramm}

Den diesjährigen Ehrenpreis der GFS e.V. für wissenschaftliche Leistungen erhielt Professor Dr. Michael Keusgen, der seit 2007 Dekan des Fachbereichs Pharmazie an der Universität Marburg ist, zahlreiche Forschungsarbeiten an mittelasiatischen Arzneipflanzen, insbesondere an Zwiebelgewächsen durchgeführt hat und sowohl an neuen pharmazeutischen Nutzungen als auch neuen Analysenmethoden arbeitet. Hervorzuheben sei auch seine Mitarbeit in Ausschüssen des Arzneibuchs und seine Tätigkeit als Vorsitzender der Gemeinsamen Expertenkommission des BVL/BfArM zur Abgrenzung von Arzneimitteln und

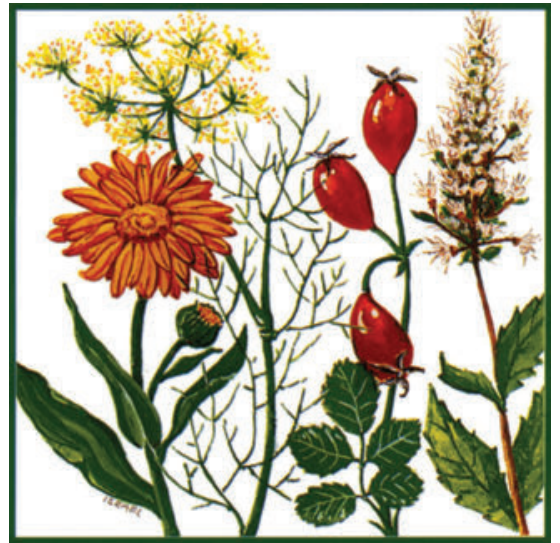

-Abb. 3 Logo von Saluplanta e. V. - die veranstaltende Gesellschaft der Bernburger Winterseminare.

Nahrungsergänzungsmitteln, wie Dr. Bernd Hoppe, Bernburg, in seiner Laudatio betonte.

Mit dem Saluplanta-Ehrenpreis 2019 wurde Prof. Dr. Bernd Honermeier ausgezeichnet, der seit 1998 am Institut für Pflanzenbau der Universität Gießen tätig und heute dessen geschäftsführender Direktor ist. Seine zahlreichen, in über 330 Veröffentlichungen beschriebenen Forschungsarbeiten beziehen sich u.a. auf Langzeiteffekte von Fruchtfolge und Düngung sowie die Qualität von Arzneipflanzen.

Wie in jedem Jahr nutzten die Teilnehmer bei der längst zur Tradition gewordenen Abendveranstaltung im Tagungssaal und zwischen den Vorträgen bei der Posterbegehung die Möglichkeit, das seit Etablierung des Bernburger Winterseminars vor knapp 30 Jahren bestehende Netzwerk aus Vertretern des Anbaus und der Arzneipflanzenverarbeitenden Industrie, aus Behörden, Hochschulen und anderen wissenschaftlichen Einrichtungen weiter auszubauen.

Dr. Barbara Steinhoff

steinhoff@phytotherapie.de 